\title{
CAN DISPOSITIONAL ESSENCES GROUND THE LAWS OF NATURE?
}

\author{
Richard Corry
}

This is an Accepted Manuscript of an article published by Taylor \& Francis Group in the Australasian Journal of Philosophy on 22 Mar 2010, available online at: http://www.tandfonline.com/10.1080/00048401003660325

A dispositional property is a tendency, or potency, to manifest some characteristic behaviour in some appropriate context. The mainstream view in the 20th Century was that such properties are to be explained in terms of more fundamental non-dispositional properties, together with the laws of nature. In the last few decades, however, a rival view has become popular. According to the rival view, some properties are essentially dispositional in nature, and the laws of nature are to be explained in terms of these fundamental dispositions. The supposed ability of fundamental dispositions to ground natural laws is one of the most attractive features of the dispositional essentialist position. In this paper, however, I cast doubt on the ability of dispositional essences to ground the laws of nature. In particular I argue that the dispositional essentialist position is not able to coherently respond - sympathetically or otherwise - to Cartwright's challenge that there are no true general laws of nature.

\section{Introduction}

During the last Century, analytic philosophy has been largely dominated by a neo-Humean metaphysic, according to which there are no necessary connections between distinct entities. David Lewis, for example, tells us that 'anything can coexist with anything else... Likewise, anything can fail to coexist with anything else' [1986: 88]. In particular, any combination of fundamental properties can be had by an object, and the properties of one object do not restrict the properties that can be had by any wholly distinct object. Thus the neo-Humean views fundamental properties as purely categorical-they do no more than categorise their possessors. It is only once we add laws of nature to the world that the fundamental properties of an object determine its dispositions to behave in certain ways under certain conditions. With different laws, the very same properties could give rise to very different behaviours. This neo-Humean metaphysic lies at the basis of both the regularity view of laws (championed by Mill [1947], Ramsey [1978], and Lewis [1973]) and the nomic necessitation view of laws (championed by David Armstrong [1983], Michael Tooley [1977], and Fred Dretske [1977]). For a long time these two views have been the only games in town. 
The last few decades, however, have seen the rise of an alternative, neo-Aristotelian ${ }^{1}$ view of properties that has come to be known as dispositional essentialism (see, for example, Popper [1957], Harré and Madden [1975], Shoemaker [1980], Ellis and Lierse [1994], Ellis [2001], Molnar [2003], Heil [2005], and Bird [2007]). According to this view, at least some fundamental properties are essentially dispositional in nature. That is, they are powers or potencies to behave in certain ways under certain conditions. So, for example, to have a positive electric charge just is to have the power to attract negatively charged objects and repel other positively charged objects. Dispositional essentialism brings with it a new approach to the laws of nature. Unlike neo-Humeanism, which analyses dispositional properties in terms of categorical properties plus the laws of nature, dispositional essentialism sees dispositional properties as fundamental, and seeks to analyse the laws of nature in terms of them. ${ }^{2}$ Indeed, one of the major motivations for dispositional essentialism is the thought that, unlike neo-Humeanism, it can provide a plausible and unified metaphysical grounding for the laws of nature (this connection between dispositions and laws has been championed by Swoyer [1982], Bostock [2001], Kistler [2002], and Bird [2007]).

Personally, I find dispositional essentialism very attractive, and I think there is something to the thought that it may provide a grounding for the laws of nature. Unfortunately, however, there is a major obstacle in the path from dispositional essences to the laws of nature, and the point of this paper is to make this obstacle clear. The problem stems from the fact that dispositional essentialism implies that the fundamental laws of nature must be strict, not ceteris paribus. This implication contradicts Nancy Cartwright's [1999] claim that all laws of nature are ceteris paribus. I do not endorse Cartwright's claim, rather the problem is that the dispositional essentialist account of laws is not compatible with Cartwright's position, and nor is it compatible with the alternative position which holds that the fundamental laws can be strict even in light of Cartwright's considerations. In Section 2, I briefly outline the derivation of laws from dispositions as it is presented in Alexander Bird's book Nature's Metaphysics: Laws and Properties [2007]. I chose this source as I believe it to be the most detailed and carefully argued account of the relation between dispositional essences and laws of nature to date, however I expect my arguments will generalise to all dispositional essentialist accounts. In Section 3 I argue that dispositional essentialism implies that the fundamental laws of nature must be strict, not ceteris paribus. Section 4 briefly rehearses Cartwright's arguments that laws are not strict. In Sections 5 and 6, I consider two possible responses to the tension and suggest that they fail.

\section{From Dispositions to Laws}

A disposition is a tendency, a capacity, a power, or potentiality, to manifest a certain behaviour given an appropriate stimulus. Thus there seems to be a deep relation between

\footnotetext{
${ }^{1}$ This terminology is from Jacobs [2007].

${ }^{2}$ For a classic neo-Humean analysis of dispositions, see Lewis [1997]
} 
the possession of a disposition and the truth of some kind of subjunctive conditional linking stimulus conditions to manifestations. This thought underlies the simple conditional analysis of dispositions which states that:

$x$ is disposed to manifest $M$ in response to stimulus $S$ iff

were $x$ to undergo $S x$ would yield manifestation $M$.

If $D_{(S, M)}$ is the disposition to $M$ under stimulus $S$, then the simple conditional analysis can be symbolised thus:

$$
D_{(S, M)} x \leftrightarrow(S x \square \longrightarrow M x)
$$

Bird argues that if (CA) is true then it is necessarily true. Thus we are led to the following simple characterisation of the nature of the disposition $D_{(S, M)}$ :

$$
\square\left(D_{(S, M)} x \leftrightarrow(S x \square \longrightarrow M x)\right)
$$

Now take some property $P$. If $P$ is essentially dispositional, then to possess $P$ is to possess a disposition to yield some manifestation $M$ in response to some stimulus $S$. Thus dispositional essentialism regarding property $P$ is that claim that for any object $x$,

$\left(D E_{P}\right) \quad \square\left(P x \rightarrow D_{(S, M)} x\right)$

Now, from $\left(C A_{\square}\right)$ and $\left(D E_{P}\right)$ we can derive

$$
\forall x((P x \& S x) \rightarrow M x)
$$

which is just the kind of universal generalisation that is usually taken to be described by the laws of nature. In particular, since the generalisation is grounded in the nature of the property $P$ and the necessary truth $\left(C A_{\square}\right)$, it is not accidental, and hence is a nomic generalisation (indeed the generalisation holds in all possible worlds, which might make it too non-accidental for some tastes). This derivation, says Bird, is 'the core of the dispositional essentialist explanation of laws' [2007: 46].

There is however a problem with this lovely picture: $\left(C A_{\square}\right)$ is false. As Bird himself points out $\left(C A_{\square}\right)$ is made false by the possibility of finks and antidotes. An object's disposition is said to be finkish if the object loses the disposition after the occurrence of the appropriate stimulus, but before the manifestation has had time to come about (see [Lewis 1997]). An antidote (also known as a mask) on the other hand, is something that leaves the disposition intact, but interferes with the causal chain between the disposition and its manifestation in such a way that the manifestation does not come about (see [Bird 1998]). Thus an object with a finkish disposition $D_{(S, M)}$ can receive the appropriate stimulus $S$ without manifesting $M$ because the disposition is lost when $S$ occurs. Similarly, in the presence of an antidote to $D_{(S, M)}$, an object may have the disposition and receive the appropriate stimulus $S$, yet fail to manifest $M$ because the antidote interferes with the operation of the disposition. In either case, the antecedent of $\left(C A_{\square}\right)$ is satisfied, while the consequent is false and so $\left(C A_{\square}\right)$ is false. 
Botulinum toxin, for example, is the most toxic substance known, with a mean lethal dose of around 1ng per kilo of body weight. Thus a tablet containing 100mg of the toxin is surely disposed to kill when ingested. Now consider two ways that one might come up with a treatment for the toxin. Winifred cultivates antibodies that bind to the toxin and mark it for destruction by the immune system. Following a long tradition of self-experimentation with cures for disease, she injects herself with these antibodies and then swallows the toxin. Thor, on the other hand, develops a suite of nano-machines that can very quickly repair the damage caused by the toxin (something like the 'nanomeds' featured in the movie The $H u l k)$. Like Winifred, he injects himself with the nano-machines and then swallows the toxin. Now suppose that both experiments are successful and neither Winifred nor Thor die. The tablets Winifred and Thor swallowed were no different to any other 100mg tablet of Botulinum toxin, and so are just as disposed to kill when ingested. But in these two cases the stimulus occurred (the tablets were ingested) without the manifestation occurring (neither Winifred nor Thor died). In Winifred's case she survived because her antibodies rendered the tablet's lethal disposition finkish. Once she swallowed the tablet, the toxin it contained was destroyed, and so its lethal disposition was removed. Thor's nano-machines, on the other hand, provided an antidote, or mask, to the tablet-the machines left the properties of the tablet itself entirely untouched but they interfered with the causal chain that would otherwise have led to Thor's death.

The falsity of $\left(C A_{\square}\right)$ may seem like a fatal blow for the dispositional essentialist account of the laws of nature, but in a lovely move Bird turns this fact to his advantage. Given that the failure of $\left(C A_{\square}\right)$ is due to the possibility of finks and antidotes, Bird [2007: 60] points out that we can replace the left-to-right half of the conditional analysis with

$\left(C A \rightarrow^{*}\right) \quad$ If $x$ has the disposition $D_{(S, M)}$ then, if $x$ were subject to $S$ and finks and antidotes to $D_{(S, M)}$ are absent, $x$ would manifest $M$.

From this and $\left(D E_{P}\right)$ we can derive the regularity:

$\left(V^{*}\right) \quad \forall x($ finks and antidotes to $D$ are absent $\rightarrow((P x \& S x) \rightarrow M x))$.

This has the form of a regularity that admits of exceptions, that is to say, $\left(V^{*}\right)$ is a ceteris paribus law. Thus the strict falsity of $\left(C A_{\square}\right)$, it seems, gives the dispositionalist account of laws more explanatory power rather than less, for dispositional essentialism can explain both strict laws and ceteris paribus laws.

\section{Fundamental Finks and Antidotes}

Having raised the possibility that dispositional essences can ground both strict and ceteris paribus laws, one might wonder whether all laws might turn out to be ceteris paribus laws (as Nancy Cartwright [1999] suggests). In particular one might wonder if there could be finks or antidotes at the fundamental level. Bird considers this question and argues that there will be no finks at the fundamental level, and that there are unlikely to be any antidotes at this level [2004; 2007]. If Bird is correct, then it follows that laws at the fundamental level will all be strict—not ceteris paribus-laws. 
In what follows I will be particularly interested in the possibility of antidotes at the fundamental level so it is worthwhile briefly rehearsing Bird's argument against this possibility (for the record, I find his argument against fundamental finks entirely convincing). Bird correctly points out that at the fundamental level antidotes cannot arise in the familiar way, through disruption of the mechanism via which a disposition produces its characteristic manifestation. For at the fundamental level there will be no lower level mechanism, and if the mechanism itself lies at the fundamental level too, then it should be re-described as a chain of mechanism-free dispositions. So the only way for antidotes to arise at the fundamental level is what he calls possibility (b):

(b) That there is no mechanism bringing about the manifestation $M$-it is brought about by $D$ and $S$ together directly. However, the further possible condition $A$ is such that $D$ and $S$ will not bring about $M$. [Bird 2007: 63]

Bird argues that 'Since we are dealing with the fundamental level, and since we have already removed the problem of multiple realizability, it might be reasonable to expect that any dispositions of this sort will suffer from relatively few antidotes.' [2007: 63] If such antidotes are few in number, he goes on to suggest, then it might not be considered too ad hoc to eliminate them in favour of antidote-free dispositions that explicitly mention the absence of these antidotes in the description of the appropriate stimulus conditions.

Bird considers the possibility of finks and antidotes at the fundamental level as a topic of intrinsic interest - perhaps it is meant to be an example of how the dispositional account of laws can cast light on wider questions in the philosophy of science. But in fact, the issue is crucial to the whole dispositionalist project. Consider what things would be like if antidotes were not rare. In fact suppose that the manifestation of some disposition is plagued by antidotes in (almost) all circumstances. In such a case the disposition will never (hardly ever) get a chance to show itself, and if a disposition is never (hardly ever) manifest, then it never (hardly ever) plays any active role in the unfolding of the world. But if that is the case then Bird's account is faced with both an epistemological and a metaphysical problem. The epistemological problem is that if a disposition is never, or hardly ever, manifest, then we will have no good reason to believe that the disposition is instantiated. But if we have no good reason to believe that a disposition is instantiated, then the disposition should play no role in our theorising about the world - in particular it should play no role in our formulation of the laws of nature. The second problem is just a metaphysical version of the first: If a disposition is hardly ever manifest, then it cannot be the source of any observed regularities in nature (even if it is in fact instantiated in all the relevant situations). Such a disposition cannot, therefore, be responsible for any observed law of nature.

The lesson of this section then, is that it is vital for the dispositional essentialist project that fundamental antidotes are not very common. Dispositional essentialism only makes sense if the fundamental laws are strict, not ceteris paribus. Thus Cartwright's claim that all laws are ceteris paribus laws poses a challenge to the dispositional essentialist. Let us turn to that challenge now. 


\section{Lying Laws: A Dilemma}

Cartwright [1983] has famously argued that the laws of physics lie. What she means is that there are very few regularities in nature of the kind described by the laws of physics. So, for example, Coulomb's Law states that between any two positively charged particles there will be a repulsive force of magnitude $\kappa_{\varepsilon} Q q / r^{2} \mathrm{~N}$. If we combine this claim with Newton's second law, $F=m a$, we can conclude that any two positively charged particles will move away from each other, with each particle accelerating at the rate $\kappa_{\varepsilon} Q q / r^{2} m \mathrm{~ms}^{-2}{ }^{3}$ But of course this is not the case. Typically there will be other forces at play that disrupt this behaviour. Two positively charged polystyrene spheres can be pushed together by a child experimenting with static electricity; the positively charged protons in the nucleus of an atom are prevented from flying apart by the strong nuclear force; Cartwright even describes a setup in which the Coulomb force between two similarly charged particles causes them to move closer together [1999: 60-61].

As Cartwright says, the mere fact that two positively charged particles are placed a distance $r$ apart tells us nothing about what will actually happen to those particles - anything at all could happen, depending on the environment in which they are placed. Because of the large diversity of relevant environments, regularities in the behaviour of such particle pairs will be exceedingly rare. In order to observe the regularity described by Coulomb's law, for example, we need to go to great trouble to screen off other influences (indeed the regularity is never strictly observed, since we cannot screen off the effects of gravity).

Cartwright's claim that there are no regularities in nature is stronger than is justified by her arguments and has been harshly criticised [Earman and Roberts 1999; Earman et al. 2002; Smith 2002; Corry 2006]. The argument involving Coulomb's Law, for example, only shows that we should not expect to observe regularities in the motions of charged particles - it remains an open possibility that there will be regularities involving other properties (more on this below). In general, Cartwright's arguments only show that there will not be strict regularities in what is often referred to as 'the course of events'. That is to say, there will be no strict regularities relating the actual values of properties such as position, velocity, mass, charge etc - the kinds of properties generally acceptable to a Humean. If we restrict our attention to properties involved in the course of events, however, Cartwright seems to be correct; we will not find strict regularities here since there will always be external factors that interfere. ${ }^{4}$ (Cartwright was not the first to point this out. The earliest explicit argument to this conclusion that I am aware of is in Russell [1913]).

${ }^{3}$ In making this move, we are, of course, failing to distinguish total and component forces. I will address this issue below.

${ }^{4}$ Even Earman and Roberts - two of Cartwright's greatest critics - accept Cartwright's claim when restricted to what they call 'phenomenological physics' [1999: 446]. By phenomenological physics they mean 'those branches of physics that aim to state correlations among more or less observable macroscopic phenomena' [1999: 473]. This is 
Now, recall that the dispositional essentialist attempts to ground the laws of nature by showing that dispositional essences entail the existence of certain regularities. So how does the dispositional account of laws mesh with Cartwright's insights? The dispositional essentialist is faced with a dilemma: either the regularities entailed by their view are regularities entirely within the course of events or these regularities involve entities and/or properties in addition to those that are found within the course of events. Unfortunately, both horns of the dilemma are sharp enough to fatally wound the dispositional essentialist project of grounding the laws of nature.

\section{The First Horn: Regularities within the Course of Events}

If the regularities entailed by dispositional essentialism relate entities or properties from within the course of events alone, then the position must deal directly with Cartwright's claim that there are no such regularities. Assuming Cartwright is right about this (as seems to be the case), we would be forced to conclude that one or more of the following is the case: (1) At least one of $\left(C A_{\square}\right)$ or $\left(D E_{P}\right)$ is false; (2) the antecedents of $\left(C A_{\square}\right)$ and/or $\left(D E_{P}\right)$ are rarely satisfied - which is to say that dispositional properties are rarely instantiated; or (3) the antecedents of $(V)$ are rarely instantiated. The second of these three options would spell doom for the dispositional essentialist project, since it would imply that dispositions play little role in the workings of the everyday world (and so would also suggest that dispositional monism is false).

So what of the first option? It seems quite attractive since, after all, everyone agrees that $\left(C A_{\square}\right)$ is false (obviously the dispositional essentialist cannot deny $\left(D E_{P}\right)$ ). As we saw, Bird says that we should replace $\left(C A_{\square}\right)$ with $\left(C A \rightarrow^{*}\right)$ which leads to the ceteris paribus law

$\left(V^{*}\right) \quad \forall x$ (finks and antidotes to $D$ are absent $\left.\rightarrow((D x \& S x) \rightarrow M x)\right)$

Indeed, the kinds of situation that Cartwright describes can naturally be understood as antidotes - they are cases where something in the environment acts to prevent the characteristic manifestation of a dispositional property, and hence disrupt the regularity that would follow from this manifestation, without removing the disposition itself. Thus what Cartwright has shown is that (within the course of events) antidotes at the fundamental level — antidotes of precisely the kind described in Bird's possibility (b) - are far from uncommon, indeed, they are ubiquitous. A pair of electrons in isolation will manifest their disposition to repel each other in accordance with Coulomb's Law, but drop a third charged particle into their vicinity, and none of the three will manifest this disposition. We must go to great trouble to shield out environmental interactions if we wish to observe the manifestation of fundamental dispositions, and even then the manifestations will only be approximate, since we cannot shield against gravitational influences. Thus, taking option (1) commits us to the view that fundamental dispositions are almost always plagued by

slightly narrower than physics restricted to the course of events, however it is only a short jump from one to the other. 
antidotes. But we saw in Section $\underline{3}$ that this option is not open to the dispositional essentialist.

Option (3) fares no better, for if the antecedents of $(V)$ are rarely instantiated, then either much of what goes on is not governed by laws (and hence is not the manifestation of a disposition), or there are a vast number of different laws (and hence different dispositions), enough to cover all possible circumstances, even though each law only applies in very limited conditions. Either way, this third option does not seem particularly attractive, and so the first horn of the dilemma is not a happy place for the dispositional essentialist.

One might object that I have ruled out this first horn of the dilemma too quickly. ${ }^{5}$ For although Cartwright argues that the laws of nature 'lie', she does not argue that there are no regularities at all. In particular, she says that under laboratory conditions - in which variables are controlled and environmental influences are carefully screened-off-we do observe regularities [1999]. It is these regularities under laboratory conditions that Cartwright believes are correctly described by the laws of physics and chemistry. What is more, Cartwright argues that the behaviour of all objects, including those in laboratory conditions, are the result of the manifestation of capacities - which are very much like the dispositions of the dispositional essentialist. Paraphrasing Cartwright then, the dispositional essentialist could argue that what happens in laboratory conditions is that, by controlling external influences, we effectively 'unmask' the relevant dispositions and thus observe the regularities to which these dispositions give rise. Since these laboratory regularities constitute what is correct about the laws of nature, we can conclude that Laws of nature, to the extent that they are correct, are metaphysically grounded in dispositions. In short, it seems that Cartwright's claims about lying laws are compatible with dispositional essentialism (as ought to be the case given that Cartwright is something very akin to a dispositional essentialist herself).

I have a suspicion that not many dispositional essentialists will be happy to take this kind of Cartwrightian position, but even if they are happy to accept the position, they ought not be. The problem is that the position leaves us wondering why it is that what we learn in the laboratory can be of any use at all in the real world. If the laws lie about the real world, how is it that we are justified in using them to help us build bridges, send spacecraft to Mars, or advise people not to jump of tall cliffs? One might respond that there is no particular problem for the dispositional essentialist here: the problem is one that everyone must face once we accept that regularities in nature are extremely rare. There is some truth in this response (which suggests to me that everyone, not just dispositional essentialists, ought reject Cartwright's claim that there are no regularities in nature), but I do think there is a particular problem for the dispositional essentialist here, for the dispositional essentialist

${ }^{5}$ Indeed the objection that follows was put to me by an anonymous referee and by a member of the audience at the conference of the Australasian Association of Philosophy in Melbourne 2009 (of course, for all I know, these may be the same person). 
metaphysics seems to undermine any possibility of an answer to the question (at least while we stick to the first horn of the dilemma and restrict manifestations to the course of events).

For suppose that, by careful experimentation, we learn that under laboratory conditionswith external influences screened-off - two electrons placed in proximity to each other will always accelerate away from each other. Following the Cartwrightian line, we may conclude that this behaviour is due to the operation of a dispositional property $D$ which reveals itself because it has been unmasked. But what of the behaviour of electrons in nonlaboratory conditions (or even in different laboratory conditions)? We know that electrons do not always accelerate away from each other, so what is the metaphysical basis of this different behaviour? There are three possibilities: (i) the behaviour of electrons in nonlaboratory conditions is also the result of their possessing $D$; (ii) the behaviour of electrons in non-laboratory conditions is the result of their possessing dispositional properties other than $D$; and (iii) the behaviour of electrons in non-laboratory conditions is not the result of the operation of dispositional properties at all.

Options (ii) and (iii) undermine our justification for applying what we learn in the laboratory to real-world situations, (or for that matter, to different laboratory situations). For if an electron's behaviour in particular laboratory conditions is due to its possession of property $D$, while its behaviour in other situations is not, then learning about its behaviour in these laboratory conditions will not tell us anything useful about how the electron will behave other situations. ${ }^{6}$

Option (i) is all that is left. If a dispositional essentialist is going to embrace a Cartwrightian position, then the only way she can justify our practice of applying the laws we learn in the laboratory to other situations (even in the expectation that they are only approximately correct) is to claim that the very same dispositional property is at play in these other situations. But this would require that the dispositional property $D$ can give rise to very different behaviours in different circumstances. It is for this sort of reason that Cartwright [1999: 56, 94] distinguishes capacities — which she says are open ended and can give rise to any number of behaviours - from dispositions - which she says are tied to particular manifestations in a one-to-one manner. Cartwright's notion of 'capacities' is very similar to the notion of a multi-track disposition: one that can have different manifestations in different circumstances. Indeed multi-track dispositions seem to fit the bill just as well.

Unfortunately, the very variability of the capacities or multi-track dispositions which make option (i) viable also make it deeply problematic. For starters there has been some suggestion that multi-track dispositions at the fundamental level are somehow illegitimate [Bird 2007: 21-24]. But even if one is not against multi-track dispositions in principle, the

${ }^{6}$ Unless there is some systematic relationship between the disposition D and the relevant dispositions in other situations. But if this is the case then we essentially have another law of nature that relates these dispositions, and the problems just arise again: What is the metaphysical grounding of the law, and how do we learn about it? 
kind of multi-track disposition required here seems particularly problematic. For the disposition will have to have very different manifestations in different situations: sometimes it might be manifest as an acceleration towards other electrons, sometimes as an acceleration away from them, sometimes as an acceleration in parallel with them, and sometimes as no acceleration at all. Similarly there are an endless number of relevantly different situations, and, particularly troubling, there is no relevant continuity among these different situations: the addition of a new particle to the situation is a discrete matter, and can make a correspondingly non-continuous difference in the manifestation. This variability and non-continuity leads to two problems, again one metaphysical and one epistemic. The metaphysical problem is that given this extreme variability and noncontinuity, there is little justification for calling all the different behaviours manifestations of the same disposition. It is more plausible to say that we have a number of single-track dispositions at play, which leads us back to option (ii). The epistemic problem is that if a disposition can give rise to such varied behaviour in different contexts, then we surely have no justification for using our knowledge of how the disposition works in one situation to make predictions in another.

All, in all, the first horn of the dilemma is not a happy home for the dispositional essentialist.

\section{The Second Horn: Going Beyond the Course of Events}

The second horn of the dilemma claims that the fundamental dispositions really do give rise to strict, not ceteris paribus, laws of nature. However we avoid Cartwright's arguments by claiming that the regularities described by these laws are not regularities in the course of events, they involve something beyond the course of events. The usual culprits for this 'something extra' are component forces. ${ }^{7}$ Proponents of this response to Cartwright include Creary [1981], Forster [1988a, b], Earman and Roberts [1999], Earman et al. [2002], Smith [2002], and Corry [2006, 2009]. As I have argued elsewhere [Corry 2009], this response is not metaphysically neutral; one must add component forces to one's ontology, and these are items that do not fit neatly into any standard ontological category. The benefits of this approach, however, are many. For the law describing the component force between a pair of charged particles only takes into account properties of, and relations between, the two particles themselves. It is thus irrelevant whether or not the pair is part of a more complex system. This fact has a number of nice consequences: (1) it means that there is no place for outside interference in the law, so we need not worry about the law being disrupted in the kind of way Cartwright describes; (2) the fact that the component force law remains constant from one context to another explains why it is that we can use knowledge gained in one context to make predictions in another; (3) in particular, the fact

${ }^{7}$ The total force on a particle is also something in addition to the course of events, and so might also be thought to be a candidate. But since total forces can be tied one-to-one with actual accelerations within the course of events, regularities involving total forces will be as rare as regularities in the course of events. 
that it is properties and relations of particle pairs that are important explains why the analytic method is so useful in science - we can best understand the component force law by studying particle pairs.

The natural dispositional account of a component force law would describe the component force between two particles as the manifestation of a disposition had by each of the particles. In particular, the force acting on particle $a$ is a manifestation of the disposition of particle $b$ to produce such a force in such circumstances. This disposition will always be manifest in the appropriate circumstances, regardless of the state of the environment. Hence the disposition will have no antidotes.

So far, then the component force approach seems to have solved the problem. We can explain the varied behaviour of complex systems in terms of component forces described by a component-force law. Furthermore, this component-force law can be explained as the result of antidote-free dispositions that the parts of the system have to produce component forces.

But a problem remains. We have explained the laws that describe the generation of component forces, but we have not yet discussed the laws that tell us how these component forces combine to produce an observable result. So, for example, the dispositionalist might tell us that Coulomb's Law is the result of the dispositions that charged particles posses to produce component forces. But what of Newton's Second Law, or the force composition law which tells us that the total force is the vector sum of the component forces? How does the dispositionalist explain these regularities?

It is tempting to suggest that a force of magnitude $F$ must posses (or simply be?) a disposition to produce an acceleration of $F / m$ when acting on a mass $m$. We can then explain Newton's Second Law in terms of such dispositions. But if we take this route then we are faced with exactly the problem we started out with - a component force of magnitude $F$ will not, in general, produce an acceleration of $F / m$ when acting on a mass $m$, for there will typically be other component forces at play. ${ }^{8}$

Bird [unpublished] has suggested that we can solve the problem by considering a force of magnitude $F$ to be a disposition to produce a change in acceleration equal to $F / m$. As we apply each force in turn these changes add up to produce the appropriate resultant acceleration. Since each force succeeds in producing its characteristic manifestation (a change in acceleration) the other forces are not acting as antidotes. Bird's suggestion is fine so long as forces are applied sequentially. But it will not solve the problem when two or more forces are applied simultaneously since then the subject of the forces will not change its acceleration by the amount characteristic of either force (a clear case is when the forces are equal and opposite so that there is no change in acceleration). The question then is

${ }^{8}$ Smith [2002: 253-4] makes much the same observation, and concludes that forces cannot be dispositions. 
whether simultaneous application of forces is likely to be common. Unfortunately, the answer is yes. The component forces acting on a particle will almost always vary as the particle moves (for example the gravitational force increases as two particles approach one another), so at every instant of time all of the component forces acting on that particle are likely to be updated with new ones. Since this is likely to happen to all component forces, the usual situation will be that at each instant of time, new component forces are applied simultaneously. Thus, Bird's suggestion does not solve the problem.

In this last section I have focused on force laws, but I think the problems raised here are general. We can avoid Cartwright's arguments by positing some new entity or property that is not part of the course of events but which provides a unifying consistency across changes in circumstance. But we will then need laws to tell us how these new entities interact, and the dispositionalist will, of course seek to understand these laws in terms of the dispositions possessed by the new entities. But now we can run the whole argument again on these dispositions, for Cartwright's considerations are likely to imply that these dispositions too will be subject to constant antidotes.

\section{Conclusion}

What I have presented here is not a knockdown argument against dispositional essentialism. I have not shown that there is anything wrong with the position itself; all I have done is cast doubt on one of the main motivations for the view. One might argue that even if the problem I have highlighted here is insoluble, this just puts dispositional essentialism on a par with neo-Humeanism, since neither can provide a satisfactory grounding for the laws of nature. But I am more optimistic than this. As I mentioned in the introduction, I find dispositional essentialism very attractive, and what's more, I can't help feeling that there is something right about the thought that dispositional essences can provide a metaphysical grounding for the laws of nature. Thus I remain hopeful that there will be some way to fix the problem, probably by tweaking a component-force type approach. Unfortunately I can't yet see how to do this. Perhaps you, dear reader, can help. ${ }^{9}$

\section{University of Tasmania}

\footnotetext{
${ }^{9}$ I am grateful to Alexander Bird, Toby Handfield, and anonymous referees for this journal for useful comments on earlier versions of this paper.
} 


\section{REFERENCES}

Armstrong, David 1983. What is a Law of Nature?, Cambridge: Cambridge University Press.

Bird, Alexander 1998. Dispositions and Antidotes, The Philosophical Quarterly 48/191: 227-234.

Bird, Alexander 2004. Antidotes all the way down?, Theoria 19/3: 259-269.

Bird, Alexander 2007. Nature's Metaphysics: Laws and Properties, Oxford University Press.

Bird, Alexander unpublished (2009). Presentation given to the Annual Meeting of the APA Central Division, Chicago.

Bostock, Simon 2001. The Necessity of Natural Laws, Ph.D. thesis, University of Sheffield.

Cartwright, Nancy 1983. How the Laws of Physics Lie, Oxford: Oxford University Press.

Cartwright, Nancy 1999. The Dappled World: A Study of the Boundaries of Science, Cambridge: Cambridge University Press.

Corry, Richard 2006. Causal Realism and the Laws of Nature, Philosophy of Science 73/3: 261-276.

Corry, Richard 2009. How is Scientific Analysis Possible?, in Dispositions and Causes, ed. Toby Handfield, Oxford: Oxford University Press: 158-188.

Creary, Lewis 1981. Causal Explanation and the Reality of Natural Component Forces, Pacific Philosophical Quarterly 62/2: 148-157.

Dretske, Fred 1977. Laws of Nature, Philosophy of Science 44/2: 248-268.

Earman, John and John Roberts 1999. Ceteris Paribus, There is no Problem of Provisos, Synthese 118/3: 439-478.

Earman, John, John Roberts, and Sheldon Smith 2002. Ceteris Paribus Lost, Erkenntnis 57/3: 281-301.

Ellis, Brian 2001. Scientific Essentialism, Cambridge: Cambridge University Press.

Ellis, Brian and Caroline Lierse 1994. Dispositional Essentialism, Australasian Journal of Philosophy 72/1: 27-45. 
Forster, Malcolm 1988a. The Confirmation of Common Component Causes, in PSA 1988, eds. A Fine and J Leplin, volume 1: 3-9.

Forster, Malcolm 1988b. Unification, Explanation, and the Composition of Causes in Newtonian Mechanics, Studies in History and Philosophy of Science 19/1: 55-101.

Harré, Rom and Edward H. Madden 1975. Causal Powers: A Theory of Natural Necessity, Oxford: Basil Blackwell.

Heil, John 2005. Dispositions, Synthese 144/3: 343-356.

Jacobs, Jonathan 2007. Causal Powers: A Neo-Aristotelian Metaphysic, Ph.D. thesis, Indiana University Bloomington.

Kistler, Max 2002. The Causal Criterion of Reality and the Necessity of Laws of Nature, Metaphysica 3/1: 57-86.

Lewis, David 1973. Counterfactuals, Oxford: Blackwell Publishers.

Lewis, David 1986. On the Plurality of Worlds, Oxford: Basil Blackwell.

Lewis, David 1997. Finkish Dispositions, Philosophical Quarterly 47/187: 143-158.

Mill, John Stewart 1947 (1843). A System of Logic, London: Longmans, Green and Co.

Molnar, George 2003. Powers, Oxford: Oxford University Press.

Popper, Karl 1957. The Propensity Interpretation of the Calculus of Probability, and the Quantum Theory, in Observation and Interpretation: A Symposium of Philosophers and Physicists, ed. S. Körner, London: Butterworths: 65-70.

Ramsey, Frank 1978 (1928). Foundations, London: Routledge and Kegan Paul.

Russell, Bertrand 1913. On the Notion of Cause, Proceedings of the Aristotelian Society 13: $1-26$.

Shoemaker, Sydney 1980. Causality and properties, in Time and Cause: Essays Presented to Richard Taylor, ed. Peter van Inwagen, Dordrecht: D. Reidel Publishing: 109-136.

Smith, Sheldon 2002. Violated Laws, Ceteris Paribus Clauses, and Capacities, Synthese 130/2: 235-264.

Swoyer, Chris 1982. The Nature of Natural Laws, Australasian Journal of Philosophy 60/3: 203-223.

Tooley, Michael 1977. The Nature of Laws, Canadian Journal of Philosophy 7/4: 667-698. 